\title{
Social Networks and Interactions in Cities*
}

\author{
Robert W. Helsley ${ }^{\dagger} \quad$ Yves Zenou ${ }^{\ddagger}$
}

February 12, 2011

\begin{abstract}
We examine how interaction choices depend on the interplay of social and physical distance, and show that agents who are more central in the social network, or are located closer to the geographic center of interaction, choose higher levels of interactions in equilibrium. As a result, the level of interactivity in the economy as a whole will rise with the density of links in the social network and with the degree to which agents are clustered in physical space. When agents can choose geographic locations, there is a tendency for those who are more central in the social network to locate closer to the interaction center, leading to a form of endogenous geographic separation based on social distance. Finally, we show that the market equilibrium is not optimal because of social externalities. We determine the value of the subsidy to interactions that could support the first-best allocation as an equilibrium and show that interaction effort and the incentives for clustering are higher under the subsidy program.
\end{abstract}

Keywords: Social networks, urban-land use, Bonacich centrality.

JEL Classification: D85, R14, Z13.

\footnotetext{
${ }^{*}$ We thank the participants of the 57th Annual North American Meetings of the Regional Science Association International, in particular, Jens Suedekum and Jacques Thisse, for helpful comments. Financial support from the Tom Hedelius' and Jan Wallander's Research Foundations is gratefully acknowledged by Yves Zenou. Financial support from the Haas School of Business and the Fisher Center for Real Estate and Urban Economics is gratefully acknowledged by Robert Helsley.

${ }^{\dagger}$ Haas School of Business, University of California, Berkeley, USA. E-mail: helsley@haas.berkeley.edu.

${ }^{\ddagger}$ Stockholm University and Research Institute of Industrial Economics (IFN), Stockholm, Sweden, and GAINS. E-mail: yves.zenou@ne.su.se.
} 


\section{Introduction}

Cities exist because proximity facilitates interactions between economic agents. There are few, if any, fundamental issues in urban economics that do not hinge in some way on reciprocal action or influence between or among workers and firms. Thus, the localization of industry arises from intra-industry knowledge spillovers in Marshall (1890), while the transmission of ideas through local inter-industry interaction fosters innovation in Jacobs (1969). In fact, the face-to-face interactions that Jacobs emphasizes are believed to be so critical to cities that Gaspar and Glaeser (1997) (and others) have asked whether advances in communication and information technology might make cities obsolete. As Glaeser and Scheinkman (2001, pp. 90) note: "Cities themselves are networks and the existence, growth, and decline of urban agglomerations depend to a large extent on these interactions."

The interactions that underlie the formation of urban areas are also important in other contexts. Following Romer $(1986,1990)$, Lucas (1988) views the local interactions that lead to knowledge spillovers as an important component of the process of endogenous economic growth. Non-market interactions also figure prominently in contemporary studies of urban crime (Glaeser, Sacerdote and Scheinkman, 1996; Verdier and Zenou, 2004), earnings and unemployment (Topa, 2001, CalvóArmengol and Jackson, 2004; Moretti, 2004; Bayer, Ross and Topa, 2008; Zenou, 2009), peer effects in education (de Bartolome, 1990; Benabou, 1993; Epple and Romano, 1998), local human capital externalities and the persistence of inequality (Benabou, 1996, and Durlauf, 1996) and civic engagement and prosperity (Putnam, 1993).

While there is broad agreement that nonmarket interactions are essential to cities and important for economic performance more broadly, the mechanisms through which local interactions generate external effects are not well understood. The dominant paradigm lies in models of spatial interaction, which assume that knowledge, or some other source of increasing returns, arises as a by-product of the production marketable goods. The level of the externality that is available to a particular firm or worker depends on its location relative to the source of the external effect the spillover is assumed to attenuate with distance - and on the spatial arrangement of economic activity. There is a rich literature (whose keystones include Beckmann, 1976; Fujita and Ogawa, 1980; and Lucas and Rossi-Hansberg, 2002) ${ }^{1}$ that examines how such spatial externalities influence the location of firms and households, urban density patterns, and productivity. There is also a substantial empirical literature (including Jaffee, Tratjenberg and Henderson, 1993; Rosenthal and Strange, 2003, 2008; and Argazi and Henderson, 2008) demonstrating that knowledge spillovers do in fact attenuate with distance. Finally, there are more specific models that treat part of the in-

\footnotetext{
${ }^{1}$ See Fujita and Thisse (2002) for a literature review.
} 
teraction process as endogenous. For example, Glaeser (1999) examines a model in which random contacts influence skill acquisition, while Helsley and Strange (2004) consider a model in which randomly matched agents choose whether and how to exchange knowledge.

This paper uses recent results from the theory of social networks to open the black box of local nonmarket interactions. We consider a population of agents who have positions within a social network and locations in a geographic space. As in Goyal (2007) and Jackson (2008), we use the tools of graph theory to model the social network. In this model the value of interaction effort increases with the efforts of others with whom one has direct links in the social network. As in Helsley and Strange (2007) and Zenou (2011), all interactions take place at a point in space, the interaction center. We examine how interaction choices depend on the interplay of social and physical distance, and show that agents who are more central in the social network, or are located closer to interaction center, choose higher levels of interactions in equilibrium. As a result, the level of interactivity in the economy as a whole will rise with the density of links in the social network and with the degree to which agents are clustered in physical space. When agents can choose geographic locations, there is a tendency for those who are more central in the social network to locate closer to the interaction center, leading to a form of endogenous geographic separation based on social distance. Finally, we show that the market equilibrium is not optimal because of social externalities. We determine the value of the subsidy to interactions that could support the first-best allocation as an equilibrium, and show that interaction effort and the incentives for clustering are higher under the subsidy program.

There is a growing interest in theoretical models of peer effects and social networks (see e.g. Akerlof, 1997; Glaeser, Sacerdote and Scheinkman, 1996; Ballester, Calvó-Armengol and Zenou, 2006; Calvó-Armengol, Patacchini and Zenou, 2009). However, there are very few papers that consider the interaction of social and physical distance. Brueckner, Thisse and Zenou (2002), Helsley and Strange (2007), Brueckner and Largey (2008) and Zenou (2011) are exceptions. However, in these models the social network is not explicitly modeled. ${ }^{2}$ To the best of our knowledge, this is the first model that combines an explicit analysis of social networks with an explicit analysis of geographic location.

The paper is organized as follows. Section 2 presents the basic model of interaction with social and physical distance, and solves for equilibrium interaction patterns. Section 3 extends the model to consider location choice and shows that agents who are more central in the social network will tend to locate closer to the center of interactions, ceteris paribus. Section 4 considers efficient interaction patterns and policies that will support the optimum as an equilibrium. Section

\footnotetext{
${ }^{2}$ See Ioannides (2011, Chap. 5) who reviews the literature on social interactions and urban economics.
} 
5 discusses our results and concludes.

\section{Equilibrium interactions with exogenous location}

\section{$2.1 \quad$ The model}

\subsubsection{Summary}

There are $n$ agents in the economy, each of whom benefits from interacting with others. The effort that an agent devotes to interactions, and the benefit that is subsequently received, depends on the agent's position in a social network and on the agent's location relative to an exogenous interaction center. Agents who are more central in the social network choose a higher level of effort, ceteris paribus, as do agents who are located closer to the interaction center. The model examines how position in the social network and geographic location combine to determine an equilibrium level of interactivity for each agent and for the economy as a whole.

\subsubsection{Locations and the social network}

The geography consists of two locations, a center, where all interactions occur, and a periphery. All agents are located in either the center or the periphery. The distance between the center and the periphery is normalized to one. Thus, letting $x_{i}$ represent the location of agent $i$, defined as her distance from the interaction center, we have $x_{i} \in\{0,1\}, \forall i=1,2, \ldots, n$. In this section we assume that locations are exogenous; location choice is considered in Section 3.

The social space is a network. A network $g$ is a set of $e x$ ante identical agents $N=\{1, \ldots, n\}$, $n \geq 2$, and a set of links or direct connections between them. These connections influence the benefit that an agent receives from interactions, in a manner that is made precise below. The adjacency matrix $\mathbf{G}=\left[g_{i j}\right]$ keeps track of the direct connections in the network. By definition, agents $i$ and $j$ are directly connected if and only if $g_{i j}=1$; otherwise, $g_{i j}=0$. We assume that if $g_{i j}=1$, then $g_{j i}=1$, so the network is undirected. ${ }^{3}$ By convention, $g_{i i}=0$. $\mathbf{G}$ is thus a square $(0,1)$ symmetric matrix with zeros on its diagonal.

\footnotetext{
${ }^{3}$ Our model can be extended to allow for directed networks (i.e. non-symmetric relationships) and weighted links in a straightforward way.
} 


\subsubsection{Preferences}

Consumers derive utility from a numeraire good $z$ and interactions with others according to the transferrable utility function

$$
U_{i}\left(v_{i}, \mathbf{v}_{-\mathbf{i}}, g\right)=z_{i}+u_{i}\left(v_{i}, \mathbf{v}_{-\mathbf{i}}, g\right)
$$

where $v_{i}$ is the number of visits that agent $i$ makes to the center, $\mathbf{v}_{-\mathbf{i}}$ is the corresponding vector of visits for the other $n-1$ agents, and $u_{i}\left(v_{i}, \mathbf{v}_{-\mathbf{i}}, g\right)$ is the subutility function of interactions. Thus, utility depends on the visit choice of agent $i$, the visit choices of other agents and on agent $i$ 's position in the social network $g$. We imagine that each visit results in one interaction, so that the aggregate number of visits is a measure of aggregate interactivity. For tractability, we assume that the subutility function takes the linear quadratic form

$$
u_{i}\left(v_{i}, \mathbf{v}_{-\mathbf{i}}, g\right)=\alpha v_{i}-\frac{1}{2} v_{i}^{2}+\theta \sum_{j=1}^{n} g_{i j} v_{i} v_{j},
$$

where $\alpha>0$ and $\theta>0$ (the roles of these parameters will become clear shortly). Equation (2) imposes additional structure on the interdependence between agents; under (2) the utility of agent $i$ depends on her own visit choice and on the visit choices of the agents with whom she is directly connected in the network, i.e., those for whom $g_{i j}=1$.

Agents located in the periphery must travel to the center to interact with others. Letting $y$ represent income and $t$ represent marginal transport cost, budget balance implies that expenditure on the numeraire is

$$
z_{i}=y-t x_{i} v_{i}
$$

Using this expression to substitute for $z_{i}$ in (1), and using (2), gives

$$
U_{i}\left(v_{i}, \mathbf{v}_{-\mathbf{i}}, g\right)=y+\alpha_{i} v_{i}-\frac{1}{2} v_{i}^{2}+\theta \sum_{j=1}^{n} g_{i j} v_{i} v_{j},
$$

where $\alpha_{i}=\alpha-t x_{i}$. We assume $\alpha>t$, so that $\alpha_{i}>0, \forall x_{i} \in\{0,1\}$ and hence $\forall i=1,2, \ldots n$. Note from (4) that utility is concave in own visits, $\frac{\partial^{2} U_{i}}{\partial v_{i}^{2}}=-1$. Note also that the marginal utility of $v_{i}$ is increasing in the visits of another with whom $i$ is directly connected, $\frac{\partial^{2} U_{i}}{\partial v_{i} \partial v_{j}}=\theta$, for $g_{i j}=1$. Thus, $v_{i}$ and $v_{j}$ are strategic complements from $i$ 's perspective when $g_{i j}=1$. Each agent $i$ chooses $v_{i}$ to maximize (4) taking the structure of the network and the visit choices of other agents as given. Before analyzing this game, we introduce a useful measure of an agent's importance in the social network. 


\subsubsection{The Katz-Bonacich network centrality measure}

There are many ways to measure the importance or centrality of an agent in a social network. For example, degree centrality measures importance by the number of direct connections that an agent has with all others, while closeness centrality measures importance by the average distance (in terms of links in the network) between an agent and all others. See Wasserman and Faust (1994) and Jackson (2008) for discussions of these, and many other, characteristics of social and economic networks. The Katz-Bonacich centrality measure (due to Katz, 1953, and Bonacich, 1987), which has proven to be extremely useful in game theoretic applications (Ballester, Calvó-Armengol and Zenou, 2006), "presumes that the power or prestige of a node is simply a weighted sum of the walks that emanate from it" (Jackson, 2008, pp. 41).

To formalize this measure, let $\mathbf{G}^{k}$ be the $k$ th power of $\mathbf{G}$, with elements $g_{i j}^{[k]}$, where $k$ is an integer. The matrix $\mathbf{G}^{k}$ keeps track of the indirect connections in the network: $g_{i j}^{[k]} \geq 0$ gives the number of walks or paths of length $k \geq 1$ from $i$ to $j$ in the network $g$. In particular, $\mathbf{G}^{0}=\mathbf{I}$. Consider the matrix $\mathbf{M}=\sum_{k=0}^{+\infty} \theta^{k} \mathbf{G}^{k}$. The elements of this matrix, $m_{i j}=\sum_{k=0}^{+\infty} \theta^{k} g_{i j}^{[k]}$, count the number of walks of all lengths from $i$ to $j$ in the network $g$, where walks of length $k$ are weighted by $\theta^{k}$. These expressions are well-defined for small enough values of $\theta{ }^{4}$ The parameter $\theta$ is a decay parameter that scales down the relative weight of longer walks. Note that, when $\mathbf{M}$ is well-defined, one can write $\mathbf{M}-\theta \mathbf{G M}=\mathbf{I}$ and hence $\mathbf{M}=[\mathbf{I}-\theta \mathbf{G}]^{-1}{ }^{5}$ The Katz-Bonacich centrality of agent $i$,

${ }^{4}$ The matrix power series $\sum_{k=0}^{+\infty} \theta^{k} \mathbf{G}^{k}$ converges if and only if

$$
\|\mathbf{G}\|<r=\lim _{k \rightarrow \infty} \inf \left|\theta^{k}\right|^{-1 / k}=\frac{1}{\theta}
$$

where $r$ is the radius of convergence and $\|\mathbf{G}\|$ is the "norm" of the matrix $\mathbf{G}$. This norm is generally taken to be the "spectral radius" of $\mathbf{G}$, written $\rho(\mathbf{G})=\max _{i}\left|\lambda_{i}\right|$, where $\lambda_{i}$ is an eigenvalue of $\mathbf{G}$. Thus, the matrix power series converges, and $\mathbf{M}$ is well-defined, for $\theta \rho(\mathbf{G})<1$. Convergence of the matrix power series constructively establishes the existence of the inverse $[\mathbf{I}-\theta \mathbf{G}]^{-1}$, where $\mathbf{I}$ is the identity matrix. The condition $\theta \rho(\mathbf{G})<1$ relates the payoff function to the network topology. When this condition holds, the local payoff interdependence $\theta$ is lower than the inverse of the spectral radius of $\mathbf{G}$, which is a measure of connectivity in the network. When this condition does not hold, existence of equilibrium becomes an issue because the strategy space is unbounded (see Ballester, Calvó-Armengol and Zenou, 2006).

${ }^{5}$ Indeed, expanding the power series gives

$$
\mathbf{M}=\mathbf{I}+\theta \mathbf{G}+\theta^{2} \mathbf{G}^{2}+\ldots,
$$

which implies,

$$
\theta \mathbf{G M}=\theta \mathbf{G}+\theta^{2} \mathbf{G}^{2}+\theta^{3} \mathbf{G}^{3}+\ldots
$$

Subtracting the latter from the former gives $\mathbf{M}-\theta \mathbf{G M}=\mathbf{I}$. 
denoted, $b_{i}(g, \theta)$ is equal to the sum of the elements of the $i$ th row of $\mathbf{M}$ :

$$
b_{i}(g, \theta)=\sum_{j=1}^{n} m_{i j}=\sum_{j=1}^{n} \sum_{k=0}^{+\infty} \theta^{k} g_{i j}^{[k]} .
$$

The Katz-Bonacich centrality of any agent is zero when the network is empty. It is also zero for $\theta=0$, and is increasing and convex in $\theta$ for $\theta>0$. For future reference, it is convenient to note that the $(n \times 1)$ vector of Katz-Bonacich centralities can be written in matrix form as

$$
\mathbf{b}(g, \theta)=\mathbf{M} \mathbf{1}=[\mathbf{I}-\theta \mathbf{G}]^{-1} \mathbf{1},
$$

where $\mathbf{1}$ is the $n$-dimensional vector of ones.

\subsection{Nash equilibrium visits and interactivity}

The first-order condition for a maximum of (4) with respect to $v_{i}$ gives the best-response function

$$
v_{i}^{*}=\alpha_{i}+\theta \sum_{j=1}^{n} g_{i j} v_{j}^{*} \quad \forall i=1,2, \ldots n .
$$

Thus, due to the linear quadratic form in (2), the optimal visit choice of agent $i$ is a linear function of the visit choices of the agents to whom $i$ is directly connected in the network. In matrix form the system in (7) becomes $\mathbf{v}=\boldsymbol{\alpha}+\theta \mathbf{G v}$, where $\boldsymbol{\alpha}$ is the $(n \times 1)$ vector of the $\alpha_{i}$ 's. Solving for $\mathbf{v}$ and using (6) gives the Nash equilibrium visit vector $\mathbf{v}^{*}$ :

$$
\mathbf{v}^{*}=[\mathbf{I}-\theta \mathbf{G}]^{-1} \boldsymbol{\alpha}=\mathbf{M} \boldsymbol{\alpha} .
$$

The Nash equilibrium visit choice of agent $i$ is

$$
v_{i}^{*}\left(x_{i}, \mathbf{x}_{-\mathbf{i}}, g\right)=\sum_{j=1}^{n} m_{i j} \alpha_{j}=\sum_{j=1}^{n} \sum_{k=0}^{+\infty} \theta^{k} g_{i j}^{[k]} \alpha_{j},
$$

where $\mathbf{x}_{-\mathbf{i}}$ is the vector of locations for the other $n-1$ agents. The expression on the right in (9) is the weighted Katz-Bonacich centrality of agent $i$, where the weight attached to the walks from $i$ to $j$ is $\alpha_{j}$. Represent this by $b_{\alpha_{i}}(g, \theta)$, so

$$
b_{\alpha_{i}}(g, \theta)=\sum_{j=1}^{n} \sum_{k=0}^{+\infty} \theta^{k} g_{i j}^{[k]} \alpha_{j} .
$$

This analysis is summarized by the following proposition: ${ }^{6}$

\footnotetext{
${ }^{6}$ For a formal proof, apply Theorem 1 in Calvó-Armengol, Patacchini and Zenou (2009) for $\beta=1, \gamma=0$, and $\lambda=\theta$.
} 
Proposition 1 (Equilibrium visits) For any networkg and for sufficiently small $\theta$, i.e. $\theta \rho(\mathbf{G})<$ 1 , there exists a unique, interior Nash equilibrium in visit choices in which the number of visits by any agent $i$ equals her weighted Katz-Bonacich centrality,

$$
v_{i}^{*}\left(x_{i}, \mathbf{x}_{-\mathbf{i}}, g\right)=b_{\alpha_{i}}(g, \theta) .
$$

The Nash equilibrium number of visits $v_{i}^{*}\left(x_{i}, \mathbf{x}_{-\mathbf{i}}, g\right)$ depends on position in the social network and geographic location. Proposition 1 implies that an agent who is more central in the social network, as measured by her Katz-Bonacich centrality, will make more visits to the interaction center in equilibrium. Intuitively, agents who are better connected have more to gain from interacting with others and so exert higher interaction effort for any vector of geographic locations.

We would like to see how the equilibrium number of visits $v_{i}^{*}\left(x_{i}, \mathbf{x}_{-\mathbf{i}}, g\right)$ varies with the different parameters of the model. It is straightforward to verify that $v_{i}^{*}\left(x_{i}, \mathbf{x}_{-\mathbf{i}}, g\right)$ increases with $\alpha$ and decreases with commuting costs $t$. It is less straighforward to analyze the relationship between $v_{i}^{*}\left(x_{i}, \mathbf{x}_{-\mathbf{i}}, g\right)$ and the intensity of social interactions $\theta$, which is also a measure of complementarity in the network. ${ }^{7}$ We have the following the result whose proof can be found in the Appendix.

Proposition 2 (Intensity of social interactions) Assume $\theta \rho(\mathbf{G})<1$. Then, for any network, an increase in the intensity of social interactions $\theta$ raises the equilibrium number of visits $v_{i}^{*}\left(x_{i}, \mathbf{x}_{-\mathbf{i}}, g\right)$ by any agent $i$.

When there are a lot of synergies from social interactions, each agent finds it desirable to visit the center more because the benefits are higher. The same intuition prevails for $\alpha$. On the contrary, when commuting costs increase, then the number of visits to the center decreases.

Let us now analyze aggregate effects. From $(9), v_{i}^{*}\left(x_{i}, \mathbf{x}_{-\mathbf{i}}, g\right)$ is non-increasing in $x_{i}$,

$$
v_{i}^{*}\left(1, \mathbf{x}_{-\mathbf{i}}, g\right)-v_{i}^{*}\left(0, \mathbf{x}_{-\mathbf{i}}, g\right)=-t m_{i i} \leq 0
$$

since $\mathbf{M}$ is a non-negative matrix. Any agent for whom $m_{i i}>0$ will make more interaction visits, or exert higher interaction effort, when located in the center rather than the periphery. In fact, reflecting the complementarity in visit choices, the equilibrium visit choice of agent $i$ is nonincreasing in the distance of any agent from the interaction center. Letting $\mathbf{x}_{-\mathbf{i k}}$ be the vector of locations for all agents except $i$ and $k$, so $\mathbf{x}_{-\mathbf{i}}=\left(x_{k}, \mathbf{x}_{-\mathbf{i k}}\right)$, we have

$$
v_{i}^{*}\left(x_{i},\left(1, \mathbf{x}_{-\mathbf{i k}}\right), g\right)-v_{i}^{*}\left(x_{i},\left(0, \mathbf{x}_{-\mathbf{i k}}\right), g\right)=-t m_{i k} \leq 0, \quad \forall k \neq i .
$$

\footnotetext{
${ }^{7}$ Recall that

$$
\frac{\partial^{2} U_{i}}{\partial v_{i} \partial v_{j}}=\theta \text { for } g_{i j}=1
$$
}


Let $V^{*}(g)$ represent the equilibrium aggregate level of visits, or, for simplicity, the equilibrium aggregate level of interactions. From (9) and (10), we have

$$
V^{*}(g)=\sum_{i=1}^{i=n} v_{i}^{*}\left(x_{i}, \mathbf{x}_{-\mathbf{i}}, g\right)=\sum_{i=1}^{i=n} b_{\alpha_{i}}(g, \theta)
$$

Consider an alternative social network $g^{\prime}, g^{\prime} \neq g$ such that for all $i, j, g_{i j}^{\prime}=1$ if $g_{i j}=1$. It is conventional to refer to $g$ and $g^{\prime}$ as nested networks, and to denote their relationship as $g \subset g^{\prime}$. As discussed in Ballester et al. (2006), the network $g^{\prime}$ has a denser structure of network links: some agents who are not directly connected in $g$ are directly connected in $g^{\prime}$. Then, given the complementarities in the network, it must be the case that equilibrium visits are weakly larger for all agents, which implies $V^{*}\left(g^{\prime}\right)>V^{*}(g)$. Similarly, (12) and (13) imply that $V^{*}(g)$ is nonincreasing in the distance of any agent from the interaction center. Thus, the more compact is the spatial arrangement of agents, the greater is the level of aggregate interactions for any network $g$. This analysis is summarized in the following proposition:

Proposition 3 (Aggregate interactions) For sufficiently small $\theta$, aggregate interactions increase with the density of network links and decrease with the distance of any agent from the interaction center.

This is an interesting result since it analyzes the relationship between network structure and aggregate interactions. It says, for example, that a star-shaped network will have fewer social interactions than a complete network because agents enjoy fewer local complementarities in the former than in the latter.

\subsection{Example}

To illustrate the previous results, consider the following star-shaped social network $g$ with three agents (i.e. $n=3$ ), where agent 1 holds a central position whereas agents 2 and 3 are peripherals:

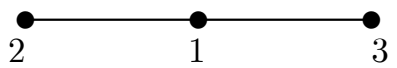

Figure 1: A star network with 3 individuals

The adjacency matrix for this social network is given by:

$$
\mathbf{G}=\left[\begin{array}{lll}
0 & 1 & 1 \\
1 & 0 & 0 \\
1 & 0 & 0
\end{array}\right]
$$


Its is a straightforward algebra exercise to compute the powers of this matrix, which are:

$$
\mathbf{G}^{2 k}=\left[\begin{array}{ccc}
2^{k} & 0 & 0 \\
0 & 2^{k-1} & 2^{k-1} \\
0 & 2^{k-1} & 2^{k-1}
\end{array}\right] \quad \text { and } \quad \mathbf{G}^{2 k+1}=\left[\begin{array}{ccc}
0 & 2^{k} & 2^{k} \\
2^{k} & 0 & 0 \\
2^{k} & 0 & 0
\end{array}\right], k \geq 1
$$

For instance, we deduce from $\mathbf{G}^{3}$ that there are exactly two walks of length three between agents 1 and 2, namely, $12 \rightarrow 21 \rightarrow 12$ and $12 \rightarrow 23 \rightarrow 32$. Obviously, there is no walk of this length (and, in general, of odd length) from any agent to herself. It is easily verified that:

$$
\mathbf{M}=[\mathbf{I}-\theta \mathbf{G}]^{-1}=\frac{1}{1-2 \theta^{2}}\left[\begin{array}{ccc}
1 & \theta & \theta \\
\theta & 1-\theta^{2} & \theta^{2} \\
\theta & \theta^{2} & 1-\theta^{2}
\end{array}\right]
$$

We can now compute the agents' centrality measures using (11). We obtain: ${ }^{8}$

$$
\left[\begin{array}{c}
v_{1}^{*} \\
v_{2}^{*} \\
v_{3}^{*}
\end{array}\right]=\left[\begin{array}{c}
b_{\alpha_{1}}(\theta, g) \\
b_{\alpha_{2}}(\theta, g) \\
b_{\alpha_{3}}(\theta, g)
\end{array}\right]=\frac{1}{1-2 \theta^{2}}\left[\begin{array}{c}
\alpha_{1}+\theta\left(\alpha_{2}+\alpha_{3}\right) \\
\theta \alpha_{1}+\left(1-\theta^{2}\right) \alpha_{2}+\theta^{2} \alpha_{3} \\
\theta \alpha_{1}+\theta^{2} \alpha_{2}+\left(1-\theta^{2}\right) \alpha_{3}
\end{array}\right]
$$

Suppose now that, for exogenous reasons, individuals 1 and 2 reside in the center, i.e., $x_{1}=x_{2}=0$ while individual 3 lives at the periphery, i.e., $x_{3}=1$. This implies that $\alpha_{1}=\alpha_{2}=\alpha$ and $\alpha_{3}=\alpha-t>0$. Thus, we now have:

$$
\left[\begin{array}{c}
v_{1}^{*} \\
v_{2}^{*} \\
v_{3}^{*}
\end{array}\right]=\frac{1}{1-2 \theta^{2}}\left[\begin{array}{c}
(1+2 \theta) \alpha-\theta t \\
(1+\theta) \alpha-\theta^{2} t \\
(1+\theta) \alpha-\left(1-\theta^{2}\right) t
\end{array}\right]
$$

It is easily verified that: ${ }^{9}$

$$
v_{1}^{*}>v_{2}^{*}>v_{3}^{*}
$$

In that case, the effort exerted by agent 1 , the most central player, is the highest one. Interestingly, even if individuals 2 and 3 have the same position in the network, because of the locational advantage in the geographical space, individual 2 has a higher weighted Katz-Bonacich centrality and thus provide higher effort than individual 3. As a result, agents located closer to the center have higher centrality $b_{\alpha_{i}}(g, \theta)$ and thus higher effort (i.e. they visit more often the center to interact with

\footnotetext{
${ }^{8}$ Note that this centrality measures are only well-defined when $\theta<1 / \sqrt{2}$ or $\theta^{2}<1 / 2$ (condition on the largest eigenvalue).

${ }^{9}$ Observe that this inequality is true because we have assumed that $\theta<1 / \sqrt{2}$ (this guarantees that the KatzBonacich centrality is well-defined) and $\alpha>t$.
} 
other people). Note that, in equilibrium, each agent $i$ 's effort is affected by the location of all other agents in the network but distant neighbors have less impact due to the decay factor $\theta$ in the Katz-Bonacich centrality.

The equilibrium aggregate level of interactions in a network is then given by:

$$
V^{*}(g)=\sum_{i=1}^{i=n} v_{i}^{*}=\frac{(3+4 \theta) \alpha-(1+\theta) t}{\left(1-2 \theta^{2}\right)}
$$

Let us now illustrate Proposition 3. Consider the network described in Figure 1 and add one link between individuals 2 and 3 so that we switch from a star-shaped network to a complete one. Suppose that we have the same geographical configuration, i.e. individuals 1 and 2 reside in the center while individual 3 lives at the periphery, i.e., $\alpha_{1}=\alpha_{2}=\alpha$ and $\alpha_{3}=\alpha-t>0$. We easily obtain: ${ }^{10}$

$$
\left[\begin{array}{c}
v_{1}^{*} \\
v_{2}^{*} \\
v_{3}^{*}
\end{array}\right]=\frac{1}{1-2 \theta^{2}-\theta}\left[\begin{array}{c}
\alpha+\alpha \theta-\theta t \\
\alpha+\alpha \theta-\theta t \\
\alpha+\alpha \theta-(1-\theta) t
\end{array}\right]
$$

Not surprisingly $v_{1}^{*}=v_{2}^{*}>v_{3}^{*}$ since all individuals have the same position in the social network but individual 3 has a "disadvantage" in the geographical space. Total activity in this network, denoted by $g^{[+23]}$, is then equal to:

$$
V^{*}\left(g^{[+23]}\right)=\frac{(3+3 \theta) \alpha-t(1-\theta+2 \theta)}{1-2 \theta^{2}-\theta}>V^{*}(g)
$$

\section{Location choice}

\subsection{Basic model}

This section extends our model of social networks and interaction to allow agents to choose between locating in the center and the periphery. To begin, and to make matters extremely simple, we suppose that there is an exogenous cost differential $C>0$ associated with the central location. Assuming that the center has more economic activity generally, this cost differential might arise from congestion effects or reflect a difference in location rent from competition among other activities for center locations. Agents choose locations to maximize net utility, that is, utility from interactions minus the exogenous location cost, taking the visits of other agents as given.

Using the best-response function (7), we can write the equilibrium utility level of agent $i$ as:

$$
U_{i}^{*}\left(v_{i}^{*}, \mathbf{v}_{-\mathbf{i}}^{*}, g\right)=y+\frac{1}{2}\left[v_{i}^{*}\left(x_{i}, \mathbf{x}_{-\mathbf{i}}, g\right)\right]^{2}=y+\frac{1}{2}\left[b_{\alpha_{i}}(g, \theta)\right]^{2}
$$

\footnotetext{
${ }^{10}$ It is easily verified that the condition on the largest eigenvalue is now given by: $\theta<1 / 2$.
} 
Thus, the gross utility difference associated with locating at the center rather than the periphery is:

$$
\begin{aligned}
\Delta U_{i}^{*} & =\frac{1}{2}\left\{\left[v_{i}^{*}\left(0, \mathbf{x}_{-\mathbf{i}}, g\right)\right]^{2}-\left[v_{i}^{*}\left(1, \mathbf{x}_{-\mathbf{i}}, g\right)\right]^{2}\right\} \\
& =\frac{1}{2}\left\{\left[v_{i}^{*}\left(0, \mathbf{x}_{-\mathbf{i}}, g\right)-v_{i}^{*}\left(1, \mathbf{x}_{-\mathbf{i}}, g\right)\right]\left[v_{i}^{*}\left(0, \mathbf{x}_{-\mathbf{i}}, g\right)+v_{i}^{*}\left(1, \mathbf{x}_{-\mathbf{i}}, g\right)\right]\right\}
\end{aligned}
$$

From the best-response function (7), we have:

$$
v_{i}^{*}\left(0, \mathbf{x}_{-\mathbf{i}}, g\right)=\alpha+\theta \sum_{j=1}^{n} g_{i j} v_{j}^{*}\left(x_{j}, \mathbf{x}_{-\mathbf{j}}, g\right),
$$

and

$$
v_{i}^{*}\left(1, \mathbf{x}_{-\mathbf{i}}, g\right)=\alpha-t+\theta \sum_{j=1}^{n} g_{i j} v_{j}^{*}\left(x_{j}, \mathbf{x}_{-\mathbf{j}}, g\right) .
$$

Substituting (18) and (19) into (17) and simplifying gives:

$$
\Delta U_{i}^{*}=t\left[v_{i}^{*}\left(0, \mathbf{x}_{-\mathbf{i}}, g\right)-\frac{t}{2}\right]>0
$$

Our earlier assumption that $\alpha>t$ implies $\Delta U_{i}^{*}>0, \forall i$. Not surprisingly, any agent has higher utility if located at the center. However, (20) shows that the utility differential is linearly increasing in $v_{i}^{*}\left(0, \mathbf{x}_{-\mathbf{i}}, g\right)$, which in turn equals agent $i$ 's weighted Bonacich centrality (for the appropriate value of $\alpha$ ) by Proposition 1 . This positive relationship between $\Delta U_{i}^{*}$ and $v_{i}^{*}$, is the key determinant of location choice: it shows that the benefit of a locating in the geographic center is greater for an agent who is more central in the social network.

To simplify notation, let $v_{i 0}^{*} \equiv v_{i}^{*}\left(0, \mathbf{x}_{-\mathbf{i}}, g\right)$. Order agents by their centrality in the social network, $v_{10}^{*} \geq v_{20}^{*} \geq \ldots v_{n 0}^{*}$, so that the weighted Katz-Bonacich centrality of agent $i$ (for the appropriate value of $\alpha$ ) is not larger than that of agent $i-1$. If $\Delta U_{n}^{*}<C$ and $\Delta U_{1}^{*}>C$, then, by continuity and the intermediate value theorem, there must exist a $v_{i 0}^{*}$ such that $t\left(v_{i 0}^{*}-t / 2\right)=C$ (see Figure 2). All agents $i \leq \widehat{i}$ will have higher net utility locating at the center; conversely, all agents $i>\widehat{i}$ will have higher net utility locating at the periphery. Thus, the bifurcation at $\widehat{i}$ generates a Nash equilibrium in geographic locations: no agent wants to deviate from this pattern, taking the visit choices of other agents as given. If $C>\Delta U_{1}^{*}$, then all agents will choose the periphery. If $C<\Delta U_{n}^{*}$, all agents will choose the center. For intermediate values, the equilibrium has the property that the most central agents locate close to the interaction center to economize on the transportation costs associated with high levels of visits or interaction effort. This analysis is summarized in the following Proposition. 
Proposition 4 (Equilibrium locations) Place agents in non-descending order based on their centralities in the social network. If the cost of locating in the center, $C$, is such that

$$
\Delta U_{n}^{*}<C<\Delta U_{1}^{*}
$$

where $\Delta U_{i}^{*}$ is the increment to utility from locating in the center for agent $i$, then there is a critical type $\hat{i}$ such that all agents who are more central in the social network than $\hat{i}$ locate in the center, while all agents who are less central than $\hat{i}$ locate in the periphery.

Proposition 4 expresses the salient relationship between position in the social network and geographic location. If participation in a social network involves costly transportation, then agents who occupy more central positions in the social network will have the most to gain from locating at the interaction center. In our model with two locations, in equilibrium agents who are most central in the social network will locate at the interaction center, while agents who are less central in the social network locate in the periphery. There is, in effect, endogenous geographic separation by position in the social network.

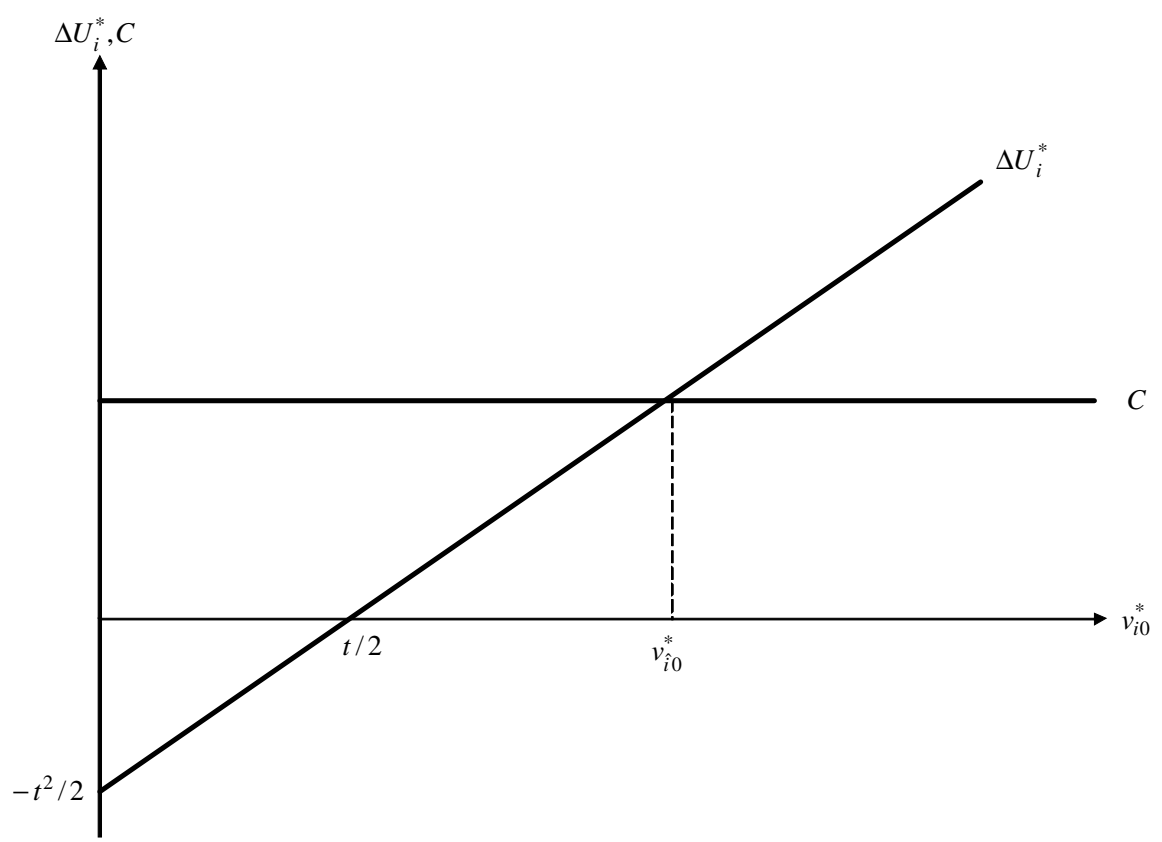

Figure 2: Nash equilibrium locations

An increase in $C$ will increase the centrality of the marginal agent $\widehat{i}$ and lead (at least weakly) to less spatial concentration at the interaction center. Similarly, an increase in marginal transport cost $t$, will cause $\Delta U_{i}$ to shift down in Figure 2. This will lead to an increase in the centrality of 
the marginal agent $\widehat{i}$ and a decrease in spatial concentration at the interaction center. Finally, an increase in $\theta$, the intensity of social interactions, will increase $v_{i 0}^{*}$ for all $i$ and thus lead to more spatial concentration in the center. This discussion is summarized in the following Proposition.

Proposition 5 (Spatial concentration in the center) An decrease in the cost of locating in the center, a decrease in marginal transport cost, or an increase in the intensity of social interactions, will lead to more spatial concentration of agents in the center.

\subsection{Example}

Let us return to the network described in Figure 1. We can now calculate the equilibrium utility (16) of each individual in the city. We have:

$$
\left[\begin{array}{c}
U_{1}^{*} \\
U_{2}^{*} \\
U_{3}^{*}
\end{array}\right]=y+\frac{1}{2\left(1-2 \theta^{2}\right)^{2}}\left[\begin{array}{c}
{\left[\alpha_{1}+\theta\left(\alpha_{2}+\alpha_{3}\right)\right]^{2}} \\
{\left[\theta \alpha_{1}+\left(1-\theta^{2}\right) \alpha_{2}+\theta^{2} \alpha_{3}\right]^{2}} \\
{\left[\theta \alpha_{1}+\theta^{2} \alpha_{2}+\left(1-\theta^{2}\right) \alpha_{3}\right]^{2}}
\end{array}\right]
$$

with $\alpha_{i}=\alpha-t x_{i}$. Let us show that there exists an equilibrium where individuals 1 and 2 live in the center and individual 3 in the periphery. Using (15) and (20), we have:

$$
\Delta U_{1}^{*}=t\left[v_{1}^{*}\left(0, \mathbf{x}_{-\mathbf{1}}, g\right)-\frac{t}{2}\right]=t\left[\frac{(1+2 \theta) \alpha-\theta t}{1-2 \theta^{2}}-\frac{t}{2}\right]
$$

where $\mathbf{x}_{-\mathbf{1}}=(0,1)$. Similarly, we have:

$$
\begin{gathered}
\Delta U_{2}^{*}=t\left[\frac{(1+\theta) \alpha-\theta^{2} t}{1-2 \theta^{2}}-\frac{t}{2}\right] \\
\Delta U_{3}^{*}=t\left[\frac{(1+\theta) \alpha-\left(1-\theta^{2}\right) t}{1-2 \theta^{2}}-\frac{t}{2}\right]
\end{gathered}
$$

We have an equilibrium with individuals 1 and 2 in the center and individual 3 in the periphery if:

$$
\Delta U_{1}^{*}>C, \Delta U_{2}^{*}>C, \text { and } \Delta U_{3}^{*}<C
$$

Since $\Delta U_{1}^{*}>\Delta U_{2}^{*}$, we need to check that:

$$
\Delta U_{3}^{*}<C<\Delta U_{2}^{*}
$$

which is condition (21) in Proposition 4. These conditions are equivalent to:

$$
(1+\theta) \alpha-\left(1-\theta^{2}\right) t<\left(1-2 \theta^{2}\right)\left(\frac{C}{t}+\frac{t}{2}\right)<(1+\theta) \alpha-\theta^{2} t
$$

Since $(1+\theta) \alpha-\left(1-\theta^{2}\right) t<(1+\theta) \alpha-\theta^{2} t$, such an equilibrium always exists if $(26)$ holds. 


\section{Welfare analysis and subsidy policies}

\subsection{First-best analysis}

We would like to see if the equilibrium outcomes are efficient in terms of social interactions. For that, the planner chooses $v_{1}, \ldots, v_{n}$ to maximize total welfare, that is:

$$
\begin{aligned}
\max _{v_{1}, \ldots, v_{n}} \mathcal{W} & =\max _{v_{1}, \ldots, v_{n}} \sum_{i=1}^{i=n} U_{i}\left(v_{i}, \mathbf{v}_{-\mathbf{i}}, g\right) \\
& =\max _{v_{1}, \ldots, v_{n}}\left\{\sum_{i=1}^{i=n}\left[y+\alpha_{i} v_{i}-\frac{1}{2} v_{i}^{2}\right]+\theta \sum_{i=1}^{i=n} \sum_{j=1}^{n} g_{i j} v_{i} v_{j}\right\}
\end{aligned}
$$

First-order condition gives for each $i=1, \ldots, n:^{11}$

$$
\alpha_{i}-v_{i}+\theta \sum_{j} g_{i j} v_{j}+\theta \sum_{j} g_{j i} v_{j}=0
$$

which implies that $\left(\text { since } g_{i j}=g_{j i}\right)^{: 12}$

$$
v_{i}^{O}=\alpha_{i}+2 \theta \sum_{j} g_{i j} v_{j}
$$

Using (7), we easily see that:

$$
v_{i}^{O}=v_{i}^{*}+\theta \sum_{j} g_{i j} v_{j}
$$

where $v_{i}^{*}$ is the Nash equilibrium number of visits given in (7). This means that there are too few visits at the Nash equilibrium as compared to the social optimum outcome. Equilibrium interaction effort is too low because each agent ignores the positive impact of a visit on the visit choices of others, that is, each agent ignores the positive externality arising from complementarity in visit choices. As a result, the market equilibrium is not efficient and the planner would like to subsidize visits to the interaction center.

\subsection{Subsidizing social interactions}

Letting $S_{i}^{O}$ denote the optimal subsidy to per visit, comparison of (24) and (25) implies:

$$
S_{i}^{O}=\theta \sum_{j} g_{i j} v_{j}
$$

\footnotetext{
${ }^{11}$ It is easily checked that there is a unique maximum for each $v_{i}$.

${ }^{12}$ The superscript $O$ refers to the "social optimum" outcome while a star refers to the "Nash equilibrium" outcome.
} 
If we add one stage before the visit game is played, the planner will announce the optimal subsidy $S_{i}^{O}$ to each agent $i$ such that:

$$
\begin{aligned}
U_{i} & =y+\left(\alpha_{i}+S_{i}^{O}\right) v_{i}-\frac{1}{2} v_{i}^{2}+\theta \sum_{j} g_{i j} v_{i} v_{j} \\
& =y+\alpha_{i} v_{i}-\frac{1}{2} v_{i}^{2}+2 \theta \sum_{j} g_{i j} v_{i} v_{j}
\end{aligned}
$$

By doing so, the planner will restore the first best. Observe that the optimal subsidy is such that

$$
\begin{aligned}
\mathbf{v}^{O} & =(\mathbf{I}-\theta \mathbf{G})^{-1}\left(\alpha_{i}+S_{i}^{O}\right) \mathbf{1} \\
& =(\mathbf{I}-2 \theta \mathbf{G})^{-1} \alpha_{i} \mathbf{1}
\end{aligned}
$$

which means that

$$
\begin{aligned}
v_{i}^{O} & =\sum_{j=1}^{n} \sum_{k=0}^{+\infty} \theta^{k} g_{i j}^{[k]}\left(\alpha_{j}+S_{j}^{O}\right) \\
& =2 \sum_{j=1}^{n} \sum_{k=0}^{+\infty} \theta^{k} g_{i j}^{[k]} \alpha_{j}
\end{aligned}
$$

and thus

$$
\begin{aligned}
U_{i}^{O} & =y+\frac{1}{2}\left[b_{\alpha+S^{O}}(\theta, g)\right]^{2} \\
& =y+\frac{1}{2}\left[b_{\alpha}(2 \theta, g)\right]^{2}
\end{aligned}
$$

What is interesting here is that the planner will give a larger subsidy to more central agents in the social network. Let us summarize our results by the following proposition.

Proposition 6 (Optimal level of social interactions) The Nash equilibrium outcome in terms of social interactions is not efficient since there are too few social interactions. If the planner proposes a subsidy $S_{i}^{O}=\theta \sum_{j} g_{i j} v_{j}$ to each individual $i$, then the first-best outcome can be restored. In that case, it is optimal for the planner to give higher subsidies to more central agents in the social network.

\subsection{Location with efficient interactions}

We would like now to investigate a constrained efficient allocation in which the planner can subsidize interactions (i.e., provide a subsidy of $S_{i}^{O}$ per visit by agent $i$ ) but cannot directly control location choices. 
From (25), we have:

$$
v_{i}^{O}-v_{i}^{*}=\theta \sum_{j} g_{i j} v_{j}>0
$$

As stated above, due to the obvious network externality, every agents makes more visits, or chooses a higher level of interaction effort, in the optimum than in the Nash equilibrium. Note that, in parallel to (16), one can write the utility level that $i$ receives in the optimum as:

$$
U_{i}^{O}\left(v_{i}^{O}, \mathbf{v}_{-\mathbf{i}}^{O}, g\right)=y+\frac{1}{2}\left[v_{i}^{O}\left(x_{i}, \mathbf{x}_{-\mathbf{i}}, g\right)\right]^{2}
$$

If agents make location decisions as before, taking the choices of others as given, then the utility differential associated with locating in the center for agent $i$ under the subsidy $S_{i}^{O}$ is (see (20)):

$$
\Delta U_{i}^{O}=t\left[v_{i}^{O}\left(0, \mathbf{x}_{-\mathbf{i}}, g\right)-\frac{t}{2}\right]>0
$$

Now consider the marginal type in the periphery $\widehat{i}$ from the equilibrium program. By construction, setting integer problems aside, for this type,

$$
\Delta U_{i}^{*}=t\left[v_{i}^{*}\left(0, \mathbf{x}_{-\mathbf{i}}, g\right)-\frac{t}{2}\right]=C
$$

Further, since $v_{i}^{O}\left(0, \mathbf{x}_{-\mathbf{i}}, g\right)>v_{i}^{*}\left(0, \mathbf{x}_{-\mathbf{i}}, g\right)$ for all $i$, we have:

$$
\Delta U_{\hat{i}}^{O}=t\left[v_{\hat{i}}^{O}\left(0, \mathbf{x}_{-\mathbf{i}}, g\right)-\frac{t}{2}\right]>C
$$

This means that under the subsidy program that supports efficient interactions, the marginal type in the periphery from the equilibrium program wants to move to the center, that is $\widehat{i}^{O}<\hat{i}^{*}$. Figure 3 displays these two solutions. Indeed, since all agents devote more effort to interacting with others under the subsidy program in (26), the incentives for clustering must be stronger under that allocation than in the Nash equilibrium. 


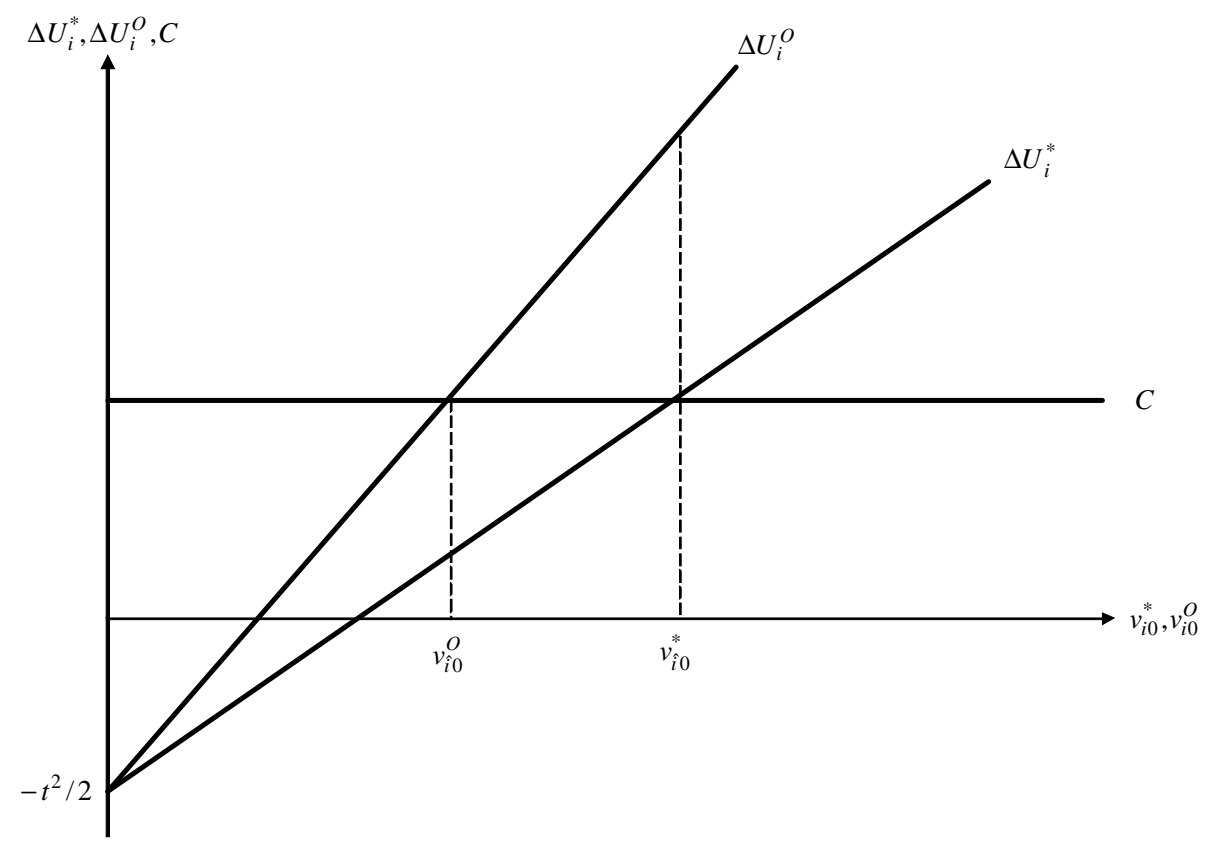

Figure 3: Nash equilibrium versus optimal location choices

Proposition 7 (Equilibrium versus optimal location choices) If the planner proposes a per visit subsidy $S_{i}^{O}$ to each individual $i$, then, compared to the Nash equilibrium location choices, more agents live in the center.

\subsection{Example}

Let us return to the network described in Figure 1 and to the example from Section 3.2. There we showed that there exists a Nash equilibrium where individuals 1 and 2 live in the center and individual 3 in the periphery if and only if (23) holds. Assume now that the planner gives a subsidy of $S_{i}^{O}=\theta \sum_{j} g_{i j} v_{j}$ per visit by agent $i$. In the context of the network described in Figure 1 , we have:

$$
S_{1}^{O}=\theta\left(v_{2}+v_{3}\right) \text { and } S_{2}^{O}=S_{3}^{O}=\theta v_{1}
$$

In that case, the Nash equilibrium generates optimum levels of visits given by: ${ }^{13}$

$$
\left[\begin{array}{c}
v_{1}^{O} \\
v_{2}^{O} \\
v_{3}^{O}
\end{array}\right]=\left[\begin{array}{c}
b_{\alpha_{1}}(2 \theta, g) \\
b_{\alpha_{2}}(2 \theta, g) \\
b_{\alpha_{3}}(2 \theta, g)
\end{array}\right]=\frac{1}{1-8 \theta^{2}}\left[\begin{array}{c}
(1+4 \theta) \alpha-2 \theta t \\
(1+2 \theta) \alpha-4 \theta^{2} t \\
(1+2 \theta) \alpha-\left(1-4 \theta^{2}\right) t
\end{array}\right]
$$

\footnotetext{
${ }^{13}$ The largest eigenvalue of $\mathbf{G}$ is still given by $\sqrt{2}$. The condition on the largest eigenvalue is, however, given by $2 \theta \rho(\mathbf{G})<1$, which is equivalent to: $4 \theta<\sqrt{2}$ or $\theta^{2}<1 / 8$.
} 
and therefore

$$
\left[\begin{array}{c}
U_{1}^{O} \\
U_{2}^{O} \\
U_{3}^{O}
\end{array}\right]=y+\frac{1}{2\left(1-8 \theta^{2}\right)^{2}}\left[\begin{array}{c}
{[(1+4 \theta) \alpha-2 \theta t]^{2}} \\
{\left[(1+2 \theta) \alpha-4 \theta^{2} t\right]^{2}} \\
{\left[(1+2 \theta) \alpha-\left(1-4 \theta^{2}\right) t\right]^{2}}
\end{array}\right]
$$

It is then easily verified that:

$$
\begin{gathered}
\Delta U_{1}^{O}=t\left[\frac{(1+4 \theta) \alpha-2 \theta t}{1-8 \theta^{2}}-\frac{t}{2}\right] \\
\Delta U_{2}^{O}=t\left[\frac{(1+2 \theta) \alpha-4 \theta^{2} t}{1-8 \theta^{2}}-\frac{t}{2}\right] \\
\Delta U_{3}^{O}=t\left[\frac{(1+2 \theta) \alpha-\left(1-4 \theta^{2}\right) t}{1-8 \theta^{2}}-\frac{t}{2}\right]
\end{gathered}
$$

Since $\Delta U_{1}^{O}>\Delta U_{2}^{O}$, the condition for this equilibrium to exist is $\Delta U_{3}^{O}<C<\Delta U_{2}^{O}$, that is:

$$
(1+2 \theta) \alpha-\left(1-4 \theta^{2}\right) t<\left(1-8 \theta^{2}\right)\left(\frac{C}{t}+\frac{t}{2}\right)<(1+2 \theta) \alpha-4 \theta^{2} t
$$

Let us now show that under the optimal subsidy policy, there can be an equilibrium for which all agents live in the center while this is not possible in the pure Nash equilibrium case. The subsidies are still given by (27) but now $\alpha_{1}=\alpha_{2}=\alpha_{3}=\alpha$. As a result,

$$
\begin{gathered}
{\left[\begin{array}{c}
v_{1}^{O} \\
v_{2}^{O} \\
v_{3}^{O}
\end{array}\right]=\frac{1}{1-8 \theta^{2}}\left[\begin{array}{c}
5 \alpha \\
\alpha(1+2 \theta) \\
\alpha(1+2 \theta)
\end{array}\right]} \\
\Delta U_{1}^{O}=t\left[\frac{5 \alpha}{1-8 \theta^{2}}-\frac{t}{2}\right] \\
\Delta U_{2}^{O}=\Delta U_{3}^{O}=t\left[\frac{\alpha(1+2 \theta)}{1-8 \theta^{2}}-\frac{t}{2}\right]
\end{gathered}
$$

The condition that guarantees that all agents live in the center is therefore $\Delta U_{2}^{O}>C$ (since $\Delta U_{1}^{O}>\Delta U_{2}^{O}$ ), that is:

$$
\frac{1}{\alpha}\left(\frac{C}{t}+\frac{t}{2}\right)<\frac{1+2 \theta}{1-8 \theta^{2}}
$$

Now, if we perform the same analysis for the Nash equilibrium case, it can be shown that the condition that guarantees that all agents live in the center is given by:

$$
\frac{1}{\alpha}\left(\frac{C}{t}+\frac{t}{2}\right)<\frac{1+\theta}{1-2 \theta^{2}}
$$


Since $\frac{1+2 \theta}{1-8 \theta^{2}}>\frac{1+\theta}{1-2 \theta^{2}}$, it is clear that (30) is much more restrictive than (29). As a result, when

$$
\frac{1+\theta}{1-2 \theta^{2}}<\frac{1}{\alpha}\left(\frac{C}{t}+\frac{t}{2}\right)<\frac{1+2 \theta}{1-8 \theta^{2}}
$$

holds, it is optimal for all three agents to choose to reside in the center under the subsidy program while at most two agents will locate in the center if no subsidy is received. This example illustrates Proposition 7. Indeed, when all agents receive a subsidy, it becomes less costly to travel to the center to interact with other agents and, as a result, they all devote more effort to interacting with others. This leads to a spatial configuration where incentives for clustering are stronger under the subsidy program than in the Nash equilibrium.

\section{Concluding remarks}

This paper provides what we believe to be the first analysis of the interaction between position in a social network and position in a geographic space, or between social and physical distance. We have developed a model in which agents who are more central in a social network, or are located closer to an interaction center, choose higher levels of interaction effort in equilibrium. As a result, the level of interactivity in the economy as a whole rises with with density of links in the social network and with the degree to which agents are clustered in physical space. When agents can choose geographic locations, there is a tendency for those who are more central in the social network to locate closer to the interaction center.

There are many potential extensions and applications of the work described here. For example, we have assumed that all interactions occur at a single, exogenous interaction center. In reality, interactions in cities occur at many sites, and whether a site becomes a focal point for interactions is of course endogenous. As in all models of complementarity, there is an interesting coordination problem in the endogenous determination of the location of an interaction center in this model. Natural applications for this analysis arise in studying the location of activities where network position seems important. For example, the most senior and presumably "best connected" business services firms in cities (law firms, consulting firms, accounting firms, and so on) appear to concentrate in downtowns, which are generally assumed to be the center of interactions for such activities. However, in other industries, like the high tech industries of the Silicon Valley, the center of interactions is actually near the geographic periphery of the metropolitan area. Finally, it might be interesting to consider relationships between various types of interactions and firm location. One might find, for example, that when internal and external interactions are complementary, firms who are more central in the social network and therefore locate close to the interaction center tend to be large, while when internal and external interactions are substitutes, firms who are more central in 
the social network tend to be small. This analysis could also be extended to study the interaction between electronic interactions, nonmarket interactions and location for firms or households with varying positions in a social network.

\section{References}

[1] Akerlof, G. (1997), "Social distance and social decisions," Econometrica 65, 1005-1027.

[2] Argazi, M. and V. Henderson (2008), "Networking off Madison avenue," Review of Economic Studies 75, 1011-1038.

[3] Ballester, C., Calvó-Armengol, A. and Y. Zenou (2006), "Who's who in networks. Wanted: The key player," Econometrica 74, 1403-1417.

[4] Bayer, P., Ross, S.L. and G. Topa (2008), "Place of work and place of residence: Informal hiring networks and labor market outcomes," Journal of Political Economy 116, 1150-1196.

[5] Beckmann, M.J. (1976), "Spatial equilibrium in the dispersed city," In: G.J. Papageorgiou (Ed.), Mathematical Land Use Theory, Lexington, MA: Lexington Books, pp. 117-125.

[6] Benabou, R. (1993), "Workings of a city: Location, education, and production," Quarterly Journal of Economics 108, 619-652.

[7] Benabou, R. (1996), "Equity and efficiency in human capital investment: The local connection," Review of Economic Studies 63, 237-264.

[8] Bonacich, P. (1987), "Power and centrality: A family of measures," American Journal of Sociology 92, 1170-1182.

[9] Brueckner, J.K. and A.G. Largey (2008), "Social interactions and urban sprawl," Journal of Urban Economics 64, 18-34.

[10] Brueckner, J.K., Thisse, J-F. and Y. Zenou (2002), "Local labor markets, job matching and urban location," International Economic Review 43, 155-171.

[11] Calvó-Armengol, A. and M.O. Jackson (2004), "The effects of social networks on employment and inequality," American Economic Review 94, 426-454.

[12] Calvó-Armengol, A., Patacchini, E., and Y. Zenou (2009), "Peer effects and social networks in education," Review of Economic Studies 76, 1239-1267. 
[13] De Bartolome, C. (1990), "Equilibrium and inefficiency in a community model with peer group effects," Journal of Political Economy 98, 110-33.

[14] Durlauf, S.N. (1996), "A theory of persistent income inequality," Journal of Economic Growth 1, 75-93.

[15] Epple, D. and R.E. Romano (1998), "Competition between private and public schools, vouchers, and peer-group effects," American Economic Review 88, 33-62.

[16] Fujita, M. and J.-F. Thisse (2002), Economics of Agglomeration. Cities, Industrial Location, and Regional Growth, Cambridge: Cambridge University Press.

[17] Fujita, M. and H. Ogawa (1980), "Equilibrium land use patterns in a nonmonocentric city," Journal of Regional Science 20, 455-475.

[18] Gaspar, J. and E.L. Glaeser (1997), "Information technology and the future of cities," Journal of Urban Economics 43, 136-156.

[19] Glaeser, E.L. (1999), "Learning in cities," Journal of Urban Economics 46, 254-277.

[20] Glaeser, E.L., Sacerdote, Bruce I. and J.A. Scheinkman (2003), "The social multiplier," Journal of the European Economic Association 1, 345-353.

[21] Glaeser, E.L. and J.A. Scheinkman (2001), "Measuring social interactions," In: S. Durlauf and P. Young (Eds.), Social Dynamics, Cambridge, MA: MIT Press, pp. 83-131.

[22] Goyal, S. (2007), Connections: An Introduction to the Economics of Networks, Princeton: Princeton University Press.

[23] Helsley, R.W. and W.C. Strange (2004), "Knowledge barter in cities," Journal of Urban Economics 56, 327-345.

[24] Helsley, R.W. and W.C. Strange (2007), "Urban interactions and spatial structure," Journal of Economic Geography 7, 119-138.

[25] Ioannides, Y.M. (2011), From Neighborhoods to Nations: The Economics of Social Interactions, forthcoming.

[26] Jackson, M.O. (2008), Social and Economic Networks, Princeton: Princeton University Press.

[27] Jacobs, J. (1969), The Economy of Cities, New York: Vintage. 
[28] Jaffe, A.B., Trajtenberg, M. and R. Henderson (1993), "Geographic localization of knowledge spillovers as evidenced by patent citations," Quarterly Journal of Economics 108, 577-598.

[29] Katz, L. (1953), "A new status index derived from sociometric analysis," Psychometrika 18, $39-43$.

[30] Lucas, R.E. (1988), "On the mechanics of economic development," Journal of Monetary Economics $22,3-42$.

[31] Lucas, R.E. and E. Rossi-Hansberg (2002), "On the internal structure of cities," Econometrica $70,1445-1476$.

[32] Marshall, A. (1890), Principles of Economics. London: Macmillan.

[33] Moretti, E. (2004), "Workers' education, spillovers, and productivity: Evidence from plantlevel production functions," American Economic Review 94, 656-690.

[34] Putnam, R.D. (1993), Bowling Alone: The Collapse and Revival of American Community, New York: Simon and Schuster.

[35] Romer, P.M. (1986), "Increasing returns and long-run growth," Journal of Political Economy 94, 1002-1037.

[36] Romer, P.M. (1990), "Endogenous technological change," Journal of Political Economy 98, S71-102.

[37] Rosenthal, S.S. and W.C. Strange (2003), "Geography, industrial organization, and agglomeration," Review of Economics and Statistics 85, 377-393.

[38] Rosenthal, S.S. and W. C. Strange (2008), "The attenuation of human capital externalities," Journal of Urban Economics 64, 373-389.

[39] Topa, G. (2001), "Social interactions, local spillovers and unemployment," Review of Economic Studies 68, 261-295.

[40] Verdier, T. and Y. Zenou (2004), "Racial beliefs, location and the causes of crime," International Economic Review 45, 727-756.

[41] Wasserman, S. and K. Faust (1994), Social Network Analysis. Methods and Applications, Cambridge: Cambridge University Press.

[42] Zenou, Y. (2009), Urban Labor Economics, Cambridge: Cambridge University Press. 
[43] Zenou, Y. (2011), "Spatial versus social mismatch: The strength of weak ties," CEPR Discussion Paper No. 8244. 


\section{Appendix}

Proof of Proposition 2. We want to study how $b_{\alpha_{i}}(g, \theta)$ varies with $\theta$. We have:

$$
\mathbf{b}_{\alpha}(g, \theta)=[\mathbf{I}-\theta \mathbf{G}]^{-1} \boldsymbol{\alpha}=\sum_{k=0}^{+\infty} \theta^{k} \mathbf{G}^{k} \boldsymbol{\alpha}
$$

and so

$$
\frac{\partial \mathbf{b}_{\alpha}(g, \theta)}{\partial \theta}=\frac{\partial}{\partial \theta}\left[\sum_{k=0}^{+\infty} \theta^{k} \mathbf{G}^{k} \boldsymbol{\alpha}\right]=\sum_{k=0}^{+\infty} k \theta^{k-1} \mathbf{G}^{k} \boldsymbol{\alpha}=\sum_{k=1}^{+\infty} k \theta^{k-1} \mathbf{G}^{k} \boldsymbol{\alpha}
$$

Since $\mathbf{G}$ and all its powers are positive matrices, and the coefficients $\theta^{k}$ increase with $\theta$, it immediately follows that the infinite series result in a matrix with all entries larger or equal than the infinite series with the initial value of $\theta$. Hence, for any fixed vector of weights $\boldsymbol{\alpha}$, the weighted Bonacich vector $\mathbf{b}_{\alpha}(g, \theta)$ increases with $\theta$. In other words, when $\theta \rho(\mathbf{G})<1$ holds, $[\mathbf{I}-\theta \mathbf{G}]^{-1}$ is well defined and

$$
\frac{\partial \mathbf{b}_{\alpha}(g, \theta)}{\partial \theta}>0
$$

and the result follows. 